\title{
Classification of Planar Anisotropic Elasticae
}

\author{
Bennett Palmer*, Álvaro Pámpano ${ }^{\dagger}$ \\ Department of Mathematics, Idaho State University, Pocatello, ID 83209, USA
}

\author{
ARTICLE INFO \\ Article History \\ Received 12 September 2019 \\ Accepted 02 December 2019 \\ Keywords \\ Anisotropic \\ elastica \\ lemniscate \\ 2000 Mathematics Subject \\ Classification \\ $53 \mathrm{~A} 04$ \\ $58 \mathrm{E} 10$
}

\begin{abstract}
We classify the anisotropic elastic curves modulo rescaling and quasi-rotation depending on one parameter for an ample family of anisotropic functionals. Several illustrations of this classification are shown at the end.
\end{abstract}

(c) 2020 The Authors. Published by Atlantis Press SARL. This is an open access article distributed under the CC BY-NC 4.0 license (http://creativecommons.org/licenses/by-nc/4.0/).

\section{INTRODUCTION}

Instances of one-dimensional continua having geometries determined by minimization of a bending energy are ubiquitous in the mathematical, physical and biological sciences. These range from models of elongated beams used in construction to the flagella of microorganisms. In our everyday experience, we encounter a myriad of fibers, wires, cables, hoses and rods whose shapes are determined by this type of variational principle. One commonly encountered aspect in the study of these continua, which we henceforth refer to as rods, is that the governing energy may be anisotropic, i.e. it is dependent on the direction of the curve, as represented by the unit tangent vector $T$. Understanding the morphology of rods is essential since the geometry is a visual manifestation of the physical properties acting on the rod.

In a recent paper [1], we developed a methodology to study the equilibria arising from minimizing a specific type of anisotropic bending energy in two and three dimensions. In this model, the Young's modulus, which measures the rod's resistance to bending, is given by a continuous periodic function of the angle the tangent makes with a fixed direction. In the two dimensional case, the equations for the extremals of this bending energy are easily integrated and all examples can be explicitly found. We recall that in the isotropic case, this is usually achieved via elliptic functions but our method does not rely on this tool.

In the isotropic case, the problem of determining the bending deformations of rods was first formulated by J. Bernoulli in 1691 .

"Corresponding author. Email: palmbenn@isu.edu

${ }^{\dagger}$ Additional information: Department of Mathematics, University of the Basque Country,

Bilbao, Bizkaia 48060, Spain
Later, D. Bernoulli, in a letter to L. Euler, suggested to study elasticae as minimizers of the bending energy. Then, L. Euler, [2] (see also the translation [3]) achieved a classification of planar elasticae into nine specific types, although some partial results were already known to J. Bernoulli. For more details about the history of (isotropic) elasticae we refer to [1] and references therein.

How is Euler's classification affected if the rod's energy is anisotropic? This is the principal question we consider. The main result of the present paper is to show that for functionals possessing an essential symmetry, all of the types in Euler's classification are present although the order in which they occur, in terms of a governing parameter, may be more complicated than in the isotropic case. Also, in the anisotropic case, there is an additional degree of freedom arising from quasi-rotations of each of the nine types.

The paper is organized as follows. In Section 2 we describe the materials and methods used for the development of the paper. In Section 3.1, we formulate the definition of anisotropic elasticae and discuss their properties. In particular, the representation formula is presented. Section 3.2 is devoted to the classification of anisotropic elasticae into nine different types. Then, in Section 3.3, we illustrate this classification for several choices of Wulff shapes. We finish with some conclusions in Section 4.

\section{MATERIALS AND METHODS}

As mentioned earlier, Euler's classification of isotropic elasticae relied heavily on the use of elliptic functions in order to represent his elasticae. In the anisotropic case, this tool is no longer applicable 
however we had previously found a relevant representation formula which can be applied to represent the curves. Since our classification theory is centered around a particular type of curve, the lemniscate, it was necessary to prove its existence. Because of the generality in the type of functional we consider, these curves could not be parameterized exactly. Instead, we rely on standard techniques of mathematical analysis to prove their existence.

Constantly, while conducting this research, it was necessary to employ computer graphics in order to generate hypotheses about how the classification would proceed and to test them. Both the Maple and Mathematica softwares were used for this purpose in an essential way. This went substantially beyond there use to generate the graphics displayed in the article.

\section{RESULTS}

\subsection{Anisotropic Elasticae}

Let $\gamma: S^{1} \rightarrow \mathbf{R}^{+}$denote a sufficiently smooth function satisfying the following convexity condition. We require that if $\theta$ denotes the usual polar angle in the plane, then

$$
\frac{1}{\mu}:=\gamma_{\theta \theta}+\gamma>0
$$

holds. The value $\gamma(\theta)$ represents the unit energy per unit length of a piece of an infinitesimal arc having tangent vector which makes an angle $\theta$ with the positive horizontal axis. The function $\mu$ represents the curvature of the plane curve given by

$$
\chi:=\theta \mapsto\left(\gamma_{\theta}-i \gamma\right) e^{i \theta},
$$

where we have identified $\mathbf{R}^{2}$ with the complex plane $\mathbf{C}$. The (convex) curve $\Omega$ defined by $\chi$ will be referred to as the Wulff shape. It was first introduced by the Ukrainian crystallographer Georg Wulff in order to model the equilibrium shape of a crystal [6]. Throughout this paper, we will say that the Wulff shape is symmetric if its curvature, $\mu$, verifies the following symmetric condition

$$
\mu\left(\frac{\pi}{2}-\theta\right)=\mu\left(\frac{\pi}{2}+\theta\right)
$$

For purposes that will be clear later, we introduce the curve $\Omega^{\perp}$ which is a clockwise rotation of $\Omega$ through an angle $\pi / 2$.

For any smooth, regular (planar) curve $C: I \rightarrow \mathbf{R}^{2}$, we denote by $T$ its unit tangent and by $N$ its unit normal with $N=J T$ where $J$ is counter-clockwise rotation by an angle $\pi / 2$. We represent $T$ by $e^{i \theta}$ and write $\gamma(\theta)$ as $\gamma(T)$ when desired. If $\kappa$ denotes the curvature of $C$, then the anisotropic curvature is defined by

$$
\lambda(s):=\frac{\kappa(s)}{\mu(\theta(s))},
$$

where $s$ represents the arc-length parameter of $C$.

As in [1], we define the anisotropic bending energy of $C$ by

$$
\mathscr{E}_{\beta}[C]:=\int_{C}\left(\lambda^{2}+\beta\right) d s
$$

where $\beta \in \mathbf{R}$. We regard $\beta$ as a Lagrange multiplier which fixes the length of the curve.

Regardless of the boundary conditions, any equilibrium curve of $\mathscr{E}_{\beta}$ must satisfy its associated Euler-Lagrange equation. We will refer to such a curve as an anisotropic elastic curve for the energy density $\gamma$. Critical points of $\mathscr{E}_{\beta}$, i.e. anisotropic elastic curves, are characterized in [1] by the conservation law

$$
\left(\frac{\lambda_{s}}{\mu}\right)^{2}+\frac{\left(\lambda^{2}-\beta\right)^{2}}{4}=p^{2}
$$

with $p \in \mathbf{R}$. If $p=0$, we have that $C$ is either a straight line or a rescaling of $\Omega^{\perp}$.

On the other hand, if $p \neq 0$, a representation formula for planar anisotropic elasticae was obtained in [1] [see formula (10)]

$$
\begin{aligned}
C=C(\theta) & = \pm \int^{\theta} \frac{e^{i \tilde{\theta}}}{\mu(\tilde{\theta}) \sqrt{2 p \sin \left(\tilde{\theta}+\theta_{o}\right)+\beta}} d \tilde{\theta} \\
& = \pm \frac{1}{\sqrt{2 p}} \int^{\theta} \frac{e^{i \tilde{\theta}}}{\mu(\tilde{\theta}) \sqrt{\sin \left(\tilde{\theta}+\theta_{0}\right)+\beta}} d \tilde{\theta}
\end{aligned}
$$

with $\beta:=\beta_{1} /(2 p)$. It will be useful to define an angle $\omega$ by

$$
\beta=-\sin \left(\frac{\pi}{2}+\omega\right)
$$

If we differentiate (5) with respect to $\theta$, we see that $e^{i \theta}$ is the unit tangent map of the curve into $S^{1}$. Clearly, $\theta$ is restricted by

$$
\frac{\pi}{2}-\omega-\theta_{0} \leq \theta \leq \frac{\pi}{2}+\omega-\theta_{0}
$$

i.e. the parameter $\beta$ controls the range of the tangent map in $S^{1}$.

Notice that, after rescaling if necessary, the constant of integration $p$ in (4) and (5), may be assumed to be $p=1 / 2$, as the following proposition shows.

Proposition 1. Let $C$ be a critical curve of $\mathscr{E}_{\beta}$. Then, any rescaling of ratio $r>0, C \rightarrow \tilde{C}=r C$, is a critical curve of $\mathscr{E}_{r^{-2} \beta}$ for $\tilde{p}^{2}=r^{-4} p^{2}$.

Proof. Let $C$ be a critical curve of $\mathscr{E}_{\beta}$ for $p \neq 0$, then the anisotropic curvature of $C, \lambda$, verifies the conservation law (4).

Now, if we apply a rescaling of ratio $r>0$ to $C$, say $\widetilde{C}(r s)=r C(s)$, then its curvature $\tilde{\kappa}$ satisfies $\kappa(s)=r \tilde{\kappa}(r s)$, where $\kappa$ denotes the curvature of $C$. Moreover, by the definition of the anisotropic curvature (2), we get that $\lambda(s)=r \tilde{\lambda}(r s)$.

Thus, substituting this in (4) we obtain

$$
\left(\frac{\lambda_{s}}{\mu}\right)^{2}+\frac{1}{4}\left(\lambda^{2}-\beta\right)^{2}=r^{4}\left[\left(\frac{\tilde{\lambda}^{\prime}}{\mu}\right)^{2}+\frac{1}{4}\left(\tilde{\lambda}^{2}-r^{-2} \beta\right)^{2}\right]=r^{4} \tilde{p}^{2}=p^{2},
$$

where we are denoting the derivative with respect to the arc-length parameter of $\widetilde{C}$ by ()$^{\prime}$. q.e.d. 
Moreover, if we apply a change of variable $\hat{\theta}=\tilde{\theta}+\theta_{0}$ in the representation formula (5), we obtain

$$
C=C(\theta)= \pm \frac{e^{-i \theta_{0}}}{\sqrt{2 p}} \int^{\theta-\theta_{0}} \frac{e^{i \hat{\theta}}}{\mu\left(\hat{\theta}-\theta_{0}\right) \sqrt{\beta+\sin (\hat{\theta})}} d \hat{\theta}
$$

This expression represents a quasi-rotation of an anisotropic planar elastica with $\theta_{0}=0$. In fact, if we rotate the Wulff shape $\Omega$ by the replacement $\gamma(\theta) \rightarrow \gamma\left(\theta-\theta_{0}\right)$, then the expression (7) above is just the rotation of an anisotropic planar elastic curve for the rotated Wulff shape. The parameter $\theta_{0}$ induces a transformation of the elasticae which is insignificant in the isotropic case.

\subsection{Classification Results}

In this section, we will classify planar anisotropic elasticae modulo rescaling and quasi-rotation for symmetric Wulff shapes. Therefore, as mentioned above, we can assume that $p=1 / 2$ and $\theta_{0}=0$, so that the representation formula for anisotropic planar elasticae (5) now reads

$$
C(\theta):= \pm \int^{\theta} \frac{e^{i \theta}}{\mu(\theta) \sqrt{\beta+\sin \theta}} d \theta
$$

The constant $\beta$ is restricted by $\beta>-1$. For these values of $\beta$, there may be isolated points where (see [1])

$$
\lambda^{2}=\beta+\sin \theta=0
$$

At these points, the curvature of $C, \kappa$, also vanishes and, therefore, they represent inflection points of the curve $C(s)$.

A particular type of lemniscate appears in Euler's classification of isotropic elastic curves given in [2]. This curve is the only closed elastica with non-constant curvature.

We begin our classification by proving the existence of an anisotropic analogue for this curve in the case that the functional possesses a symmetric Wulff shape. These examples appear whenever the inflection points of $C(s)$ happen to be double points. In the anisotropic case, however, this lemniscate may not be unique. An example is given below of a functional having three distinct anisotropic lemniscates.

We will need the following technical lemma.

Lemma 1. Let $\mu$ be any positive function. Then, the function $\tilde{\psi}:(0, \pi) \rightarrow \mathbf{R}$ defined by

$$
\tilde{\psi}(\omega):=\int_{\frac{\pi}{2}-\omega}^{\frac{\pi}{2}+\omega} \frac{\sin \theta}{\mu(\theta) \sqrt{\sin \theta-\sin \left(\frac{\pi}{2}+\omega\right)}} d \theta
$$

is continuous. Moreover, it has at least one zero in the interval $(\pi / 2, \pi)$.

Proof. We begin by proving that the function $\tilde{\psi}$ is continuous in $(0, \pi)$. For this purpose, we first choose an $\varepsilon>0$ such that $\omega+\varepsilon<\pi$. Then, after decomposing the domain in three different parts, we have that

$$
\tilde{\psi}(\omega+\varepsilon)-\tilde{\psi}(\omega):=I_{1}+I_{2}+I_{3}
$$

where

$$
\begin{gathered}
I_{1}=\int_{\frac{\pi}{2}-(\omega+\varepsilon)}^{\frac{\pi}{2}-\omega} \frac{\sin \theta}{\mu(\theta) \sqrt{\sin \theta-\sin \left(\frac{\pi}{2}+\omega+\varepsilon\right)}} d \theta, \\
I_{2}=\int_{\frac{\pi}{2}+\omega}^{\frac{\pi}{2}+\omega+\varepsilon} \frac{\sin \theta}{\mu(\theta) \sqrt{\sin \theta-\sin \left(\frac{\pi}{2}+\omega+\varepsilon\right)}} d \theta,
\end{gathered}
$$

and

$$
\begin{aligned}
& I_{3}= \\
& \int_{\frac{\pi}{2}-\omega}^{\frac{\pi}{2}+\omega} \frac{\sin \theta}{\mu(\theta)}\left(\frac{1}{\sqrt{\sin \theta-\sin \left(\frac{\pi}{2}+\omega+\varepsilon\right)}}-\frac{1}{\sqrt{\sin \theta-\sin \left(\frac{\pi}{2}+\omega\right)}}\right) d \theta .
\end{aligned}
$$

Consider the limit of the third integral, $I_{3}$, when $\mathcal{E} \searrow 0$. Note that

$$
\begin{aligned}
\left|I_{3}\right| \leq c_{3} \int_{\frac{\pi}{2}-\omega}^{\frac{\pi}{2}+\omega}\left(\frac{1}{\sqrt{\sin \theta-\sin \left(\omega+\varepsilon+\frac{\pi}{2}\right)}}-\frac{1}{\sqrt{\sin \theta-\sin \left(\omega+\frac{\pi}{2}\right)}}\right) d \theta \\
\quad \leq c_{3} \int_{\frac{\pi}{2}-\omega}^{\frac{\pi}{2}+\omega} \frac{1}{\sqrt{\sin \theta-\sin \left(\omega+\varepsilon+\frac{\pi}{2}\right)}} d \theta<\infty,
\end{aligned}
$$

where $c_{3}$ is a suitable constant. Then, since the integrand is non negative, we can apply the Dominated Convergence Theorem to obtain that $I_{3} \rightarrow 0$ as $\varepsilon \searrow 0$.

For the second integral, $I_{2}$, we make the change of variable $\theta=\pi / 2+$ $\omega+t$ to obtain

$$
\begin{aligned}
\left|I_{2}\right| & \leq c_{2} \int_{\frac{\pi}{2}+\omega}^{\frac{\pi}{2}+\omega+\varepsilon} \frac{1}{\sqrt{\sin \theta-\sin \left(\frac{\pi}{2}+\omega+\varepsilon\right)}} d \theta \\
& =c_{2} \int_{0}^{\varepsilon} \frac{1}{\sqrt{\sin \left(\frac{\pi}{2}+\omega+t\right)-\sin \left(\frac{\pi}{2}+\omega+\varepsilon\right)}} d t \\
& =c_{2} \int_{0}^{\varepsilon} \frac{1}{\sqrt{\cos (\omega+t)-\cos (\omega+\varepsilon)}} d t,
\end{aligned}
$$

where $c_{2}$ is a constant. We then use the Taylor expansion of $\cos (\omega+t)$ centered at $t=\varepsilon$,

$$
\cos (\omega+t)=\cos (\omega+\varepsilon)+\sin (\omega+\varepsilon)(\varepsilon-t)+\mathcal{O}\left([\varepsilon-t]^{2}\right)
$$

to get

$$
\frac{1}{\sqrt{\cos (\omega+t)-\cos (\omega+\varepsilon)}} \leq \tilde{c}_{2} \frac{1}{\sqrt{\varepsilon-t}}
$$


for another constant $\tilde{c}_{2}$. Hence,

$$
\left|I_{2}\right| \leq c_{2} \tilde{c}_{2} \int_{0}^{\varepsilon} \frac{1}{\sqrt{\varepsilon-t}} d t=\hat{c_{2}} \sqrt{\varepsilon} \rightarrow 0
$$

as $\mathcal{E} \searrow 0$, for a suitable constant $\hat{c}_{2}$.

Moreover, a similar argument works for the first integral $I_{1}$ and, therefore,

$$
|\tilde{\psi}(\omega+\varepsilon)-\tilde{\psi}(\omega)| \leq\left|I_{1}\right|+\left|I_{2}\right|+\left|I_{3}\right| \rightarrow 0
$$

as $\mathcal{E} \backslash 0$. Finally, note that the case $\varepsilon<0$ can be treated similarly to the case $\varepsilon>0$. This proves the continuity.

Now, we just need to check that in the interval $(\pi / 2, \pi)$ there is a change of sign, so that there exists a zero of $\tilde{\psi}$. First, we have that at $\omega=\pi / 2$, the integral $\tilde{\psi}(\pi / 2)$ simplifies to

$$
\widetilde{\psi}\left(\frac{\pi}{2}\right)=\int_{0}^{\pi} \frac{\sqrt{\sin \theta}}{\mu(\theta)} d \theta
$$

which is clearly positive.

Now take a $\delta>0$, such that $\delta<\pi / 2$, then at $\omega=\pi-\delta$ we have

$$
\tilde{\psi}(\pi-\delta)=\int_{-\frac{\pi}{2}+\delta}^{\frac{3 \pi}{2}-\delta} \frac{\sin \theta}{\mu(\theta) \sqrt{\sin \theta-\sin \left(\frac{3 \pi}{2}-\delta\right)}} d \theta
$$

which can be bounded as follows. First, notice that, after suitable change of variable

$$
\begin{aligned}
\tilde{\psi}(\pi-\delta)= & 2 \int_{-\frac{\pi}{2} \delta}^{0} \frac{\sin \theta}{\mu(\theta) \sqrt{\sin \theta-\sin \left(\frac{3 \pi}{2}-\delta\right)}} d \theta \\
& +\int_{0}^{\pi} \frac{\sin \theta}{\mu(\theta) \sqrt{\sin \theta-\sin \left(\frac{3 \pi}{2}-\delta\right)}} d \theta \\
= & J_{1}+J_{2} .
\end{aligned}
$$

Then, taking the limit as $\delta \searrow 0$, we have that the second integral above, $J_{2}$, converges to a positive number which is independent of $\delta$, but the first one goes to $-\infty$. This follows from the fact that for $\theta \in(-\pi / 2+\delta, 0), \sin \theta$ is always negative and

$$
\begin{aligned}
& \sin (\theta)-\sin \left(\frac{3 \pi}{2}-\delta\right) \\
& =\sin (\theta)-\sin \left(\frac{-\pi}{2}+\delta\right) \approx\left(\theta-\left[\frac{-\pi}{2}+\delta\right]\right) \cos \left(\frac{-\pi}{2}+\delta\right)
\end{aligned}
$$

for $\theta \approx-\pi / 2$. Combining this with the fact that $0<<\mu<<\infty$, the result follows. q.e.d.

We deduce from this the following existence result.

Theorem 1. For any symmetric Wulff shape $\Omega$, there exists a constant $\beta_{l} \in(0,1)$ such that the critical curve of $\mathscr{B}_{\beta_{l}}$ with non-constant anisotropic curvature, $\lambda$, is closed.

Proof. Let $0<\beta<1$ and denote by $C(s)$ any critical curve of $\mathscr{E}_{\beta}$ with non-constant anisotropic curvature. The inflection points of $C(s)$ are given by the solutions of (9). By (6), these points are, precisely, $\theta=\pi / 2 \pm \omega$ depending on the value of $\omega \in(\pi / 2, \pi)$.

As noticed above, we have that the closed critical curve appears whenever the inflection points are precisely the double points of the curve $C$. This means that $C(\pi / 2+\omega)=C(\pi / 2-\omega)$.

Define the (a priori complex-valued) function

$$
\psi(\omega)=-i\left[C\left(\frac{\pi}{2}+\omega\right)-C\left(\frac{\pi}{2}-\omega\right)\right] .
$$

The result follows by checking that there exists a value $\omega_{1}$ such that $\psi\left(\omega_{l}\right)=0$, since this $\omega_{l}$ would give rise to a value $\beta_{l}$ [see (6)] whose associated critical curve is closed.

Note that $\psi$ takes only real values. In fact, by using the representation formula for $C(8)$, we have that for any $\omega \in(\pi / 2, \pi)$,

$$
\psi(\omega)=\int_{\frac{\pi}{2}-\omega}^{\frac{\pi}{2}+\omega} \frac{-i e^{i \theta}}{\mu(\theta) \sqrt{\beta+\sin \theta}} d \theta=\int_{\frac{\pi}{2}-\omega}^{\frac{\pi}{2}+\omega} \frac{\sin \theta}{\mu(\theta) \sqrt{\beta+\sin \theta}} d \theta \in \mathbf{R}
$$

where the second equality is obtained since $\cos (\pi / 2-t)=-\cos (\pi / 2$ $+t)$ and $\sin (\pi / 2-t)=\sin (\pi / 2+t)$ for any value of $t$ and applying the symmetric condition on the function $\mu$ (1).

Then, it is clear that $\psi(\omega)=\tilde{\psi}(\omega)$ and, therefore, we can apply Lemma 1 to get that $\psi(\omega)$ has a zero in $(\pi / 2, \pi)$, as desired. q.e.d.

Up to quasi-rotations and rescalings there is at least one anisotropic elastica with non-constant anisotropic curvature associated to $\beta_{l} \in(0,1)$ which we will refer to as an anisotropic elastic lemniscate (For some illustrations, see Figure 1).

Now, we are in conditions to prove the classification of planar anisotropic elasticae.
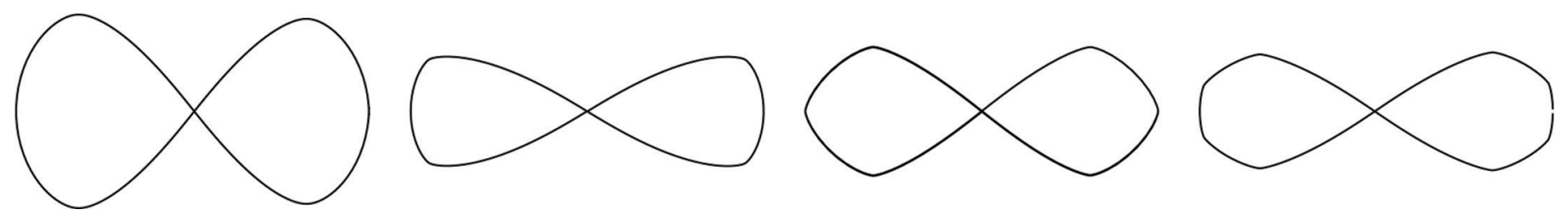

Figure 1 Lemniscate elasticae $\left(0<\beta_{l}=\beta<1\right)$. 
Theorem 2. Let $C$ be a planar anisotropic elastica for a symmetric Wulff shape $\Omega$, i.e. a critical curve of $\mathscr{E}_{\beta}$. If the anisotropic curvature of $C, \lambda$, is constant, then $C$ is either a straight line $(\lambda=0)$ or a rescaling of $\Omega^{\perp}\left(\lambda^{2}=\beta\right)$.

If $\lambda$ is non constant, we have the following families depending on the parameter $\beta>-1$ :

1. Orbit-like anisotropic elasticae, if $\beta>1$.

2. Borderline anisotropic elasticae, if $\beta=1$.

3. Wave-like anisotropic elasticae, if $-1<\beta<1$. In this case, we have the following sub-cases:

(i) Multiloop anisotropic elasticae, if $0<\beta=-\sin (\pi / 2+\omega)<1$ and $\psi(\omega)<0$.

(ii) Lemniscate anisotropic elasticae, if $0<\beta=\beta_{l}<1$.

(iii) Deep waves, if $0<\beta=-\sin (\pi / 2+\omega)<1$ and $\psi(\omega)>0$.

(iv) Rectangular anisotropic elasticae, if $\beta=0$.

(v) Shallow waves, if $-1<\beta<0$.

Proof. Let $C$ be a critical curve of $\mathscr{E}_{\beta}$ for a fixed $\beta$. The case $\lambda$ constant has been explained before, giving rise to either straight lines or rescalings of $\Omega^{\perp}$ (see Figure 2). Thus, from now on, we assume that $\lambda$ is not constant. We recall that $\theta$ represents the angle that the tangent to $C$ makes with the horizontal axis. The proof is going to be divided in terms of the different possible values for $\beta$.
We begin by considering $\beta>1$ (see Figure 3). In this case, (9) tells us that $\lambda$ never vanishes, i.e., there are no inflection points on the anisotropic elastica $C$ and, as a consequence, $\theta$ varies in the whole real line. Thus, we have orbit-like anisotropic elasticae.

If $-1<\beta \leq 1$, from (9) it is clear that at any interval of length $2 \pi$ where $\theta$ varies there are exactly two inflection points. Therefore, $\theta$ is only defined in an open interval of length smaller or equal $2 \pi$ starting at one inflection point, and finishing at the other one. This means that if we consider our anisotropic curve $C$ to start at one of those inflection points, $\theta$ varies until reaching the following inflection point, then, $\theta$ goes back again.

Take now $\beta=1$ (see Figure 4). In this particular case, the domain of definition for $\theta$ can be considered to be $(-\pi / 2,3 \pi / 2)$. Now, since $\lim _{\theta \rightarrow-\pi / 2} \lambda=\lim _{\theta \rightarrow 3 \pi / 2} \lambda=0$, we have that the curve $C$ tends to a straight line at the end points. Moreover, the tangent at the end points tends to make an angle of $-\pi / 2(3 \pi / 2$, respectively) with the horizontal axis, i.e. $C$ tends to a vertical line. This case gives rise to a single loop and, hence, it corresponds with borderline anisotropic elasticae.

Assume $0<\beta<1$. For a fixed $\beta$, we define the function

$$
\widehat{\psi}(t)=\int_{\frac{\pi}{2}-t}^{\frac{\pi}{2}+t} \frac{\sin \hat{\theta}}{\mu(\hat{\theta}) \sqrt{\beta+\sin \hat{\theta}}} d \hat{\theta} .
$$

By a similar argument as in Lemma 1, we have that above function is continuous for $t \in(0, \omega)$. Recall that $\omega$ is defined by (6) in terms of $\beta$, hence, it is a fixed value, $\omega \in(\pi / 2, \pi)$. The limit of $\widehat{\psi}(t)$ when
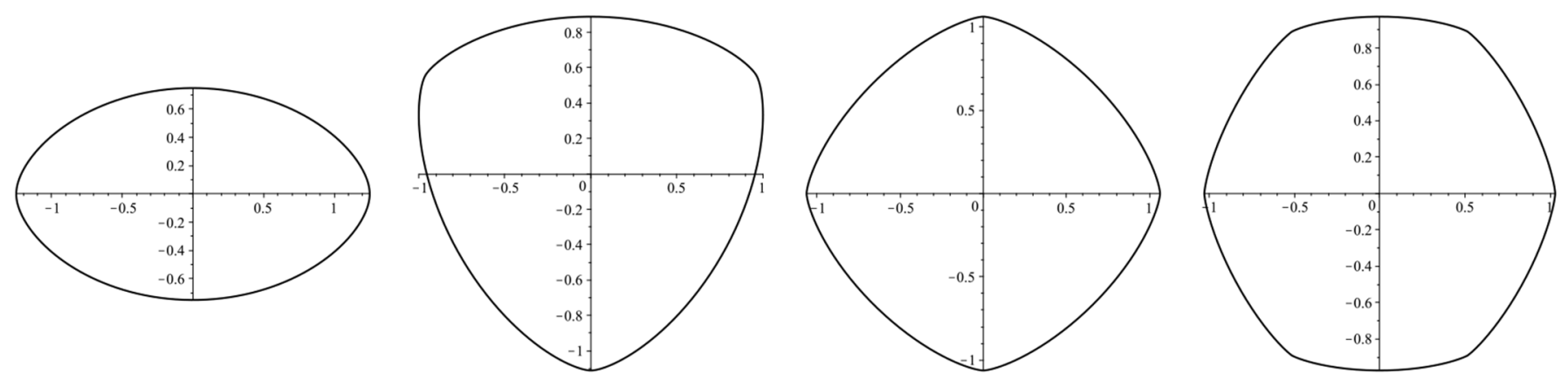

Figure 2 The Wulff shapes $\Omega_{n}$, for $n=2,3,4$ and 6. Recall that the critical points with $\lambda^{2}=\beta$ correspond to $\Omega_{n}^{\perp}$.
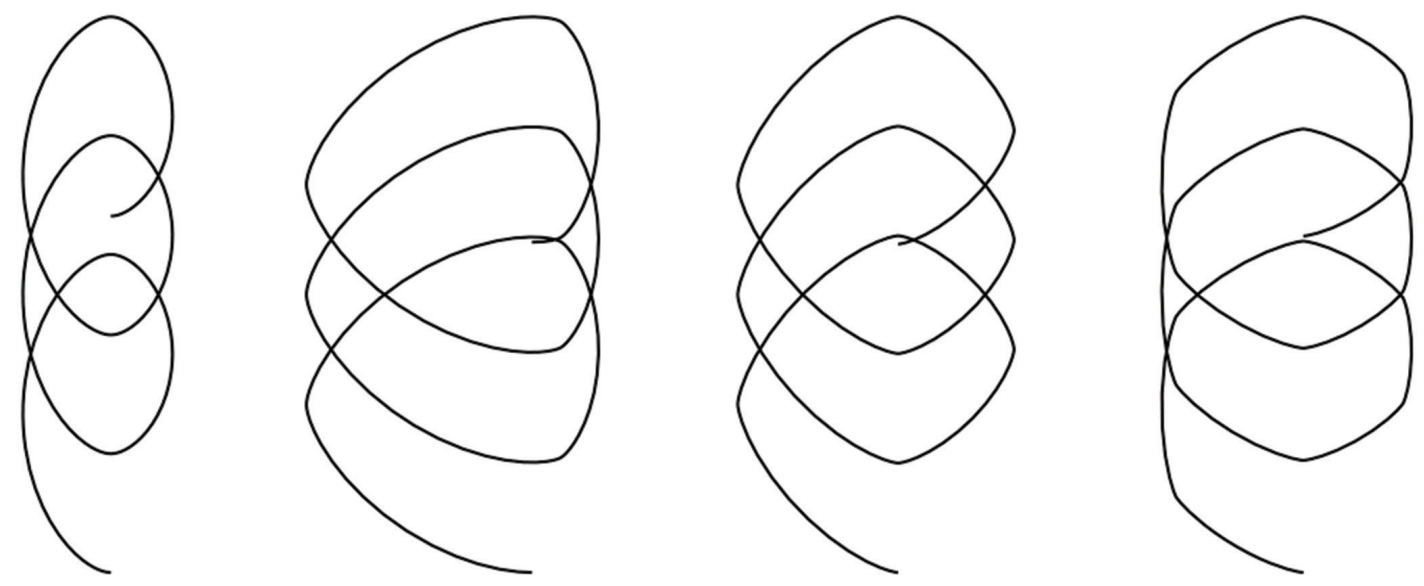

Figure 3 Orbit-like anisotropic elasticae $(\beta>1)$. 
$t \rightarrow 0$ is clearly zero; while $\widehat{\psi}(t) \rightarrow \psi(\omega)$, as $t \rightarrow \omega$. Moreover, we also have

$$
\frac{d \widehat{\psi}}{d t}(t)=\frac{2 \cos t}{\mu(\pi / 2+t) \sqrt{\beta+\cos t}}
$$

due to the symmetric condition (1). This means that $\widehat{\psi}$ increases as $t$ varies from 0 to $\pi / 2$. Then, it decreases until $t=\omega$. Thus, depending on the sign of $\psi(\omega)$, we have different sub-cases:

- Sub-case $0<\beta<1$ and $\psi(\omega)<0$ (see Figure 5). In this case, the function $\widehat{\psi}(t)$ has exactly one change of sign which means that, between any two consecutive inflection points, there is a double point, i.e. we have multiloop anisotropic elasticae.

- Sub-case $0<\beta<1$ and $\psi(\omega)=0$ (see Figure 1 ). This case occurs when $\beta=\beta$ and the associated anisotropic elastica is a lemniscate. The existence is guaranteed in Theorem 1 .

- Sub-case $0<\beta<1$ and $\psi(\omega)>0$ (see Figure 6). Contrary to the multiloop case, here, we have that $\widehat{\psi}$ is always positive and, thus, there are no self-intersections between consecutive inflection points. This gives rise to deep waves.

Now for $\beta=0$ (see Figure 7), the domain of the tangent map on $S^{1}$ is a semicircle, since $\theta \in(0, \pi)$. Hence, this corresponds with the rectangular anisotropic elasticae. Note that in this case, the anisotropic elastica cuts the vertical axis orthogonally.

Finally, for the case $-1<\beta<0$ (see Figure 8 ), the arc-length of the domain of the tangent map is smaller than $\pi$, producing shallow waves. This finishes the proof. q.e.d.
For many choices of $\gamma$, the types of elasticae vary 'monotonically' as $\beta$ decreases. However for others, we observed that this is not the case due to some oscillation in the sign of $\psi(\omega)$. In particular, Figure 9 shows three distinct lemniscates which occur for the functional having density $\gamma_{16}:=1+\cos (16 \theta) /(16)^{2}$. The Wulff shape for this functional is a smoothed 16-gon (See Section 3.3 for more details).

As a final remark, observe that the existence of anisotropic elastic lemniscate is not guaranteed if the Wulff shape does not verify the symmetry condition (1), since, in this case, $\psi(\omega) \notin \mathbf{R}$ [see (10)] and therefore the analysis made in Lemma 1 is not applicable. Indeed, consider the non-symmetric Wulff shape $\widehat{\Omega}$ defined by the following function $\mu$

$$
\frac{1}{\mu}:=1-\frac{15}{16} \sin (4 \theta) .
$$

Then, as suggested in Figure 10 there are no anisotropic lemniscates for this Wulff shape $\widehat{\Omega}$. However, the other eight types of elasticae in the classification are present in this case.

\subsection{Illustrations}

In order to obtain some illustrations of above classification, we consider the following densities. If $n$ is an even positive integer, we define

$$
\gamma_{n}=\gamma_{n}(\theta):=1+\frac{\cos (n \theta)}{n^{2}}
$$
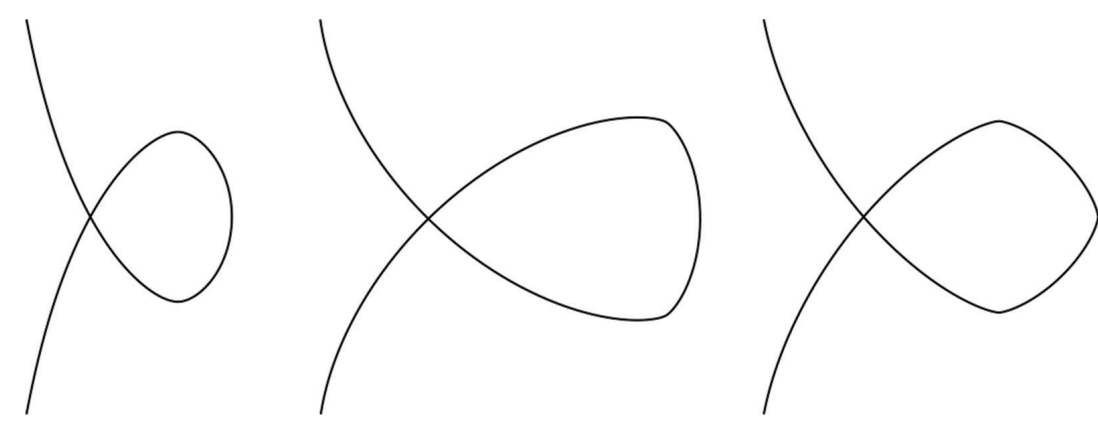

Figure $4 \mid$ Borderline anisotropic elasticae $(\beta=1)$.
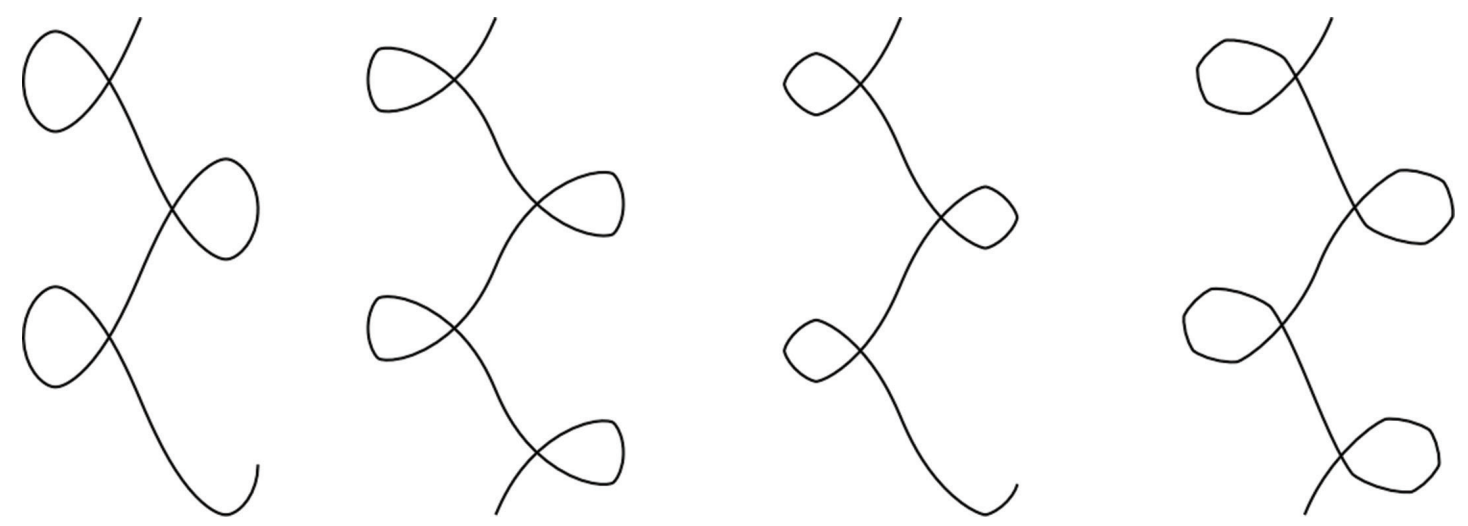

Figure 5 Multiloop anisotropic elasticae $(0<\beta<1$ and $\psi(\omega)<0)$. 

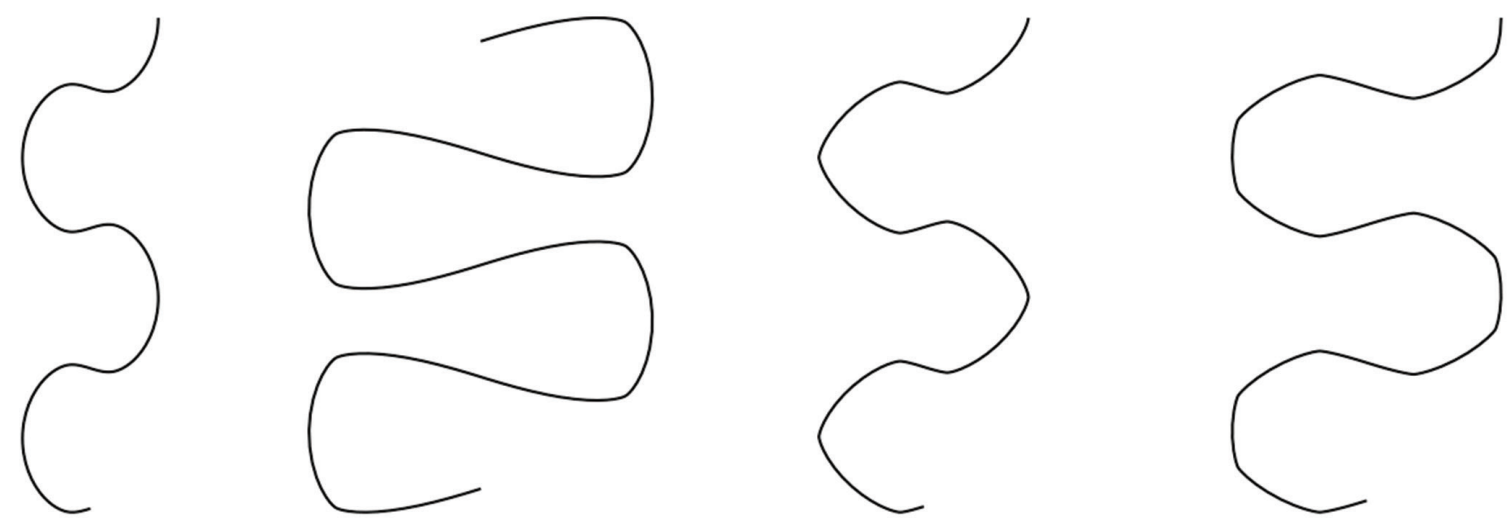

Figure $6 \mid$ Deep waves $(0<\beta<1$ and $\psi(\omega)>0)$.
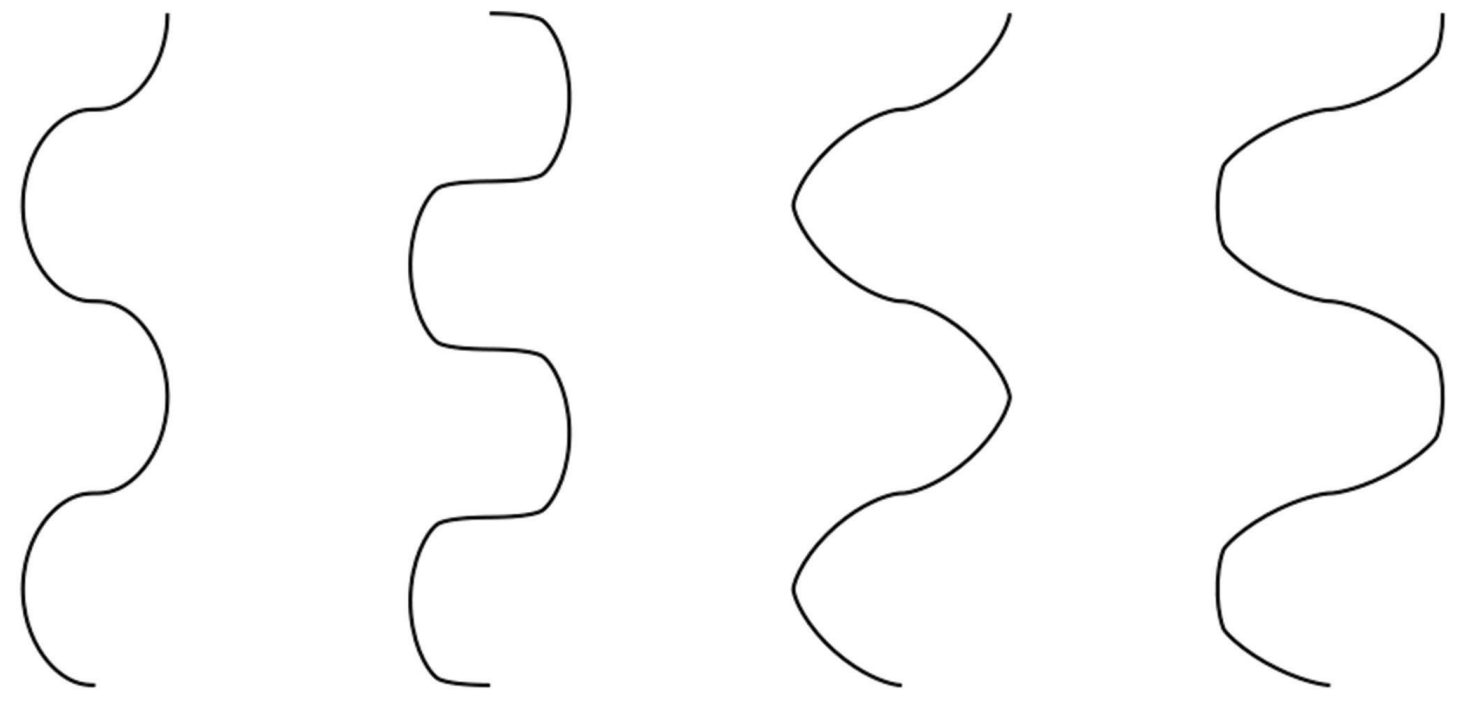

Figure 7 Rectangular anisotropic elasticae $(\beta=0)$.
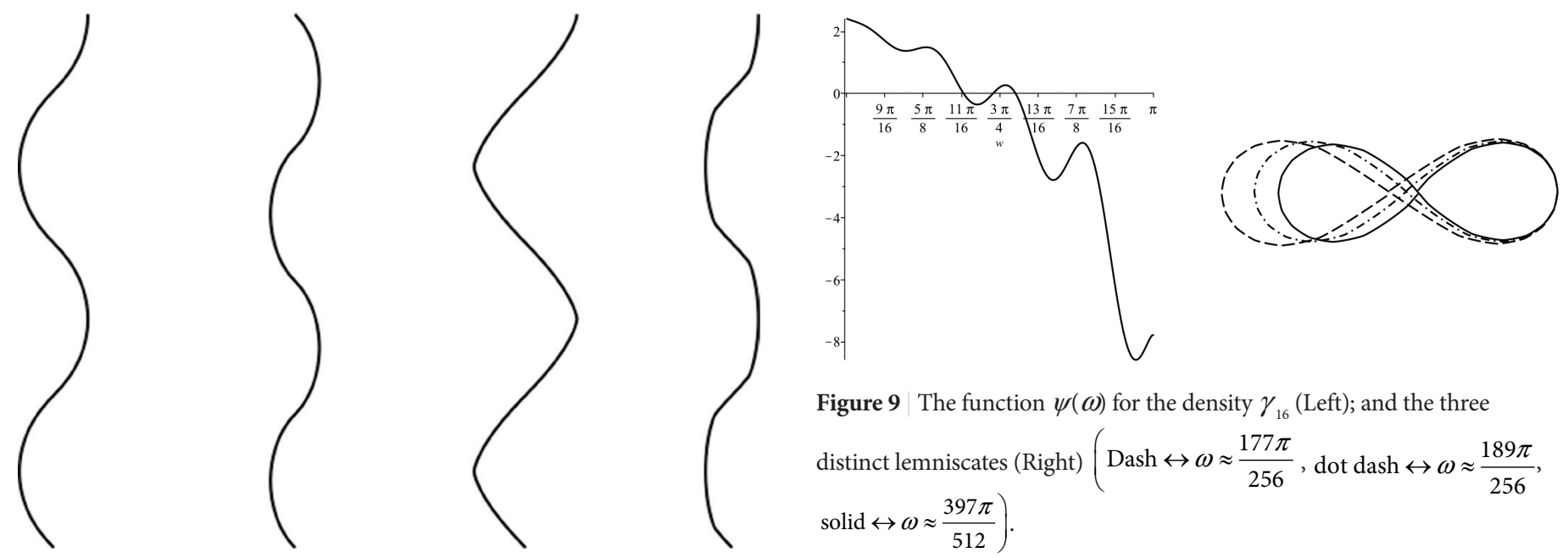

Figure 8 Shallow waves $(-1<\beta<0)$.

Figure 9 The function $\psi(\omega)$ for the density $\gamma_{16}($ Left); and the three distinct lemniscates (Right) (Dash $\leftrightarrow \omega \approx \frac{177 \pi}{256}$, dot dash $\leftrightarrow \omega \approx \frac{189 \pi}{256}$, solid $\left.\leftrightarrow \omega \approx \frac{397 \pi}{512}\right)$. 


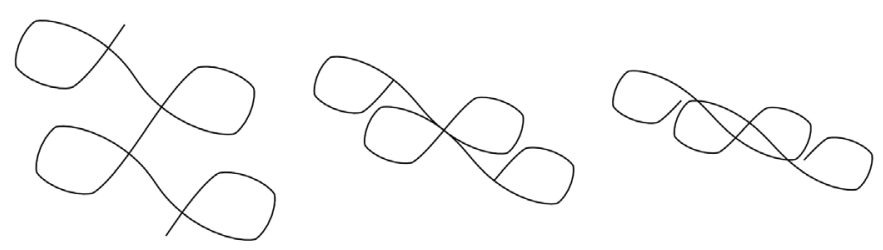

Figure 10 Evolution of anisotropic elasticae for $\widehat{\Omega}$ from multiloop (Left) to deep wave (Right). The intermediate step (Center) is not an anisotropic elastic lemniscate.
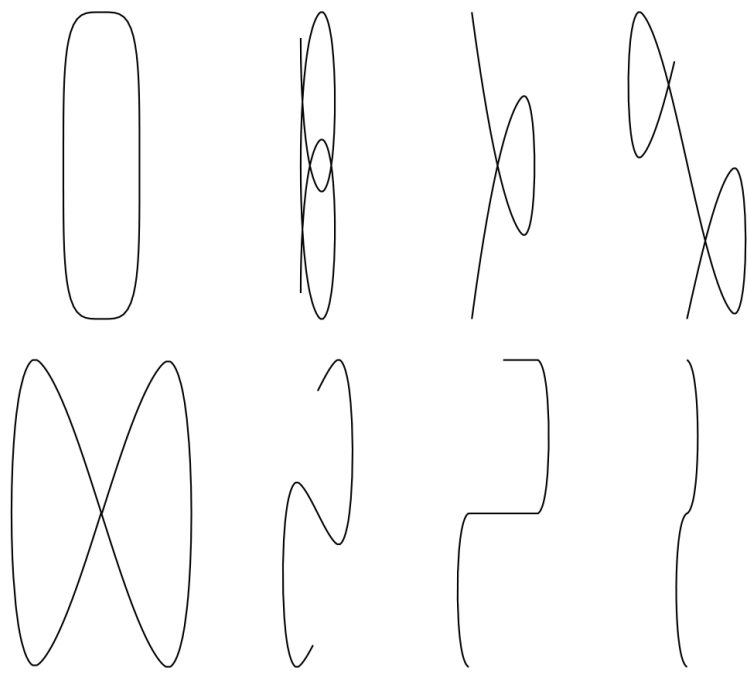

Figure 11 Anisotropic elasticae for the Wulff Shape, $\widetilde{\Omega}$, as the parameter $\beta$ decreases. The first figure represents the rotated Wulff shape, $\widetilde{\Omega}^{\perp}$.

Then,

$$
\frac{1}{\mu}=\gamma_{\theta \theta}+\gamma=1-\left(\frac{n^{2}-1}{n^{2}}\right) \cos (n \theta) .
$$

On the other hand, if $n$ is an odd positive integer, $\gamma_{n}$ is going to be

$$
\gamma_{n}=\gamma_{n}(\theta):=1+\frac{\sin (n \theta)}{n^{2}}
$$

In these cases,

$$
\frac{1}{\mu}=\gamma_{\theta \theta}+\gamma=1-\left(\frac{n^{2}-1}{n^{2}}\right) \sin (n \theta) .
$$

We will refer to the Wulff shape for the density $\gamma_{n}$ as $\Omega_{n}$. The distinction between the odd and even cases is done so that the function $\mu$ verifies the symmetric condition (1). Several illustrations of these Wulff shapes and the different types of associated anisotropic elasticae, produced using (8) are shown in Figures 1-8.

To obtain a greater variety of Wulff shapes, we will use the following construction. Take the polar coordinates $(r, \theta)$, where the radial function $r=r(\theta)$ is given by

$$
r(\theta)=\left(\left|\frac{\cos \left(\frac{m_{a} \theta}{4}\right)}{a}\right|^{n_{a}}+\left|\frac{\sin \left(\frac{m_{b} \theta}{4}\right)}{b}\right|^{n_{b}}\right)^{-1 / n}
$$

for real constants $a, b, m_{a}, m_{b}, n_{a}, n_{b}$ and $n$. This formula known as the superformula was introduced by Gielis to study naturally occurring shapes $[5,6]$. In particular, for some choices of specific values of the constants, the corresponding curve is convex, so that it can be used to generate different Wulff shapes.

We are going to choose the following constants $b=1, a=m_{a}=m_{b}$ $=n_{a}=n_{b}=n=4$ and denote by $\widetilde{\Omega}$ the corresponding Wulff shape. From Figure 11 it is clear that $\Omega$ is convex and possesses the correct symmetry. We can compute its curvature, $\mu$, using the standard formula

$$
\mu(\theta)=\frac{2\left[r^{\prime}(\theta)\right]^{2}+r^{2}(\theta)-r(\theta) r^{\prime \prime}(\theta)}{\left(\left[r^{\prime}(\theta)\right]^{2}+r^{2}(\theta)\right)^{3 / 2}} .
$$

The rotated Wulff shape together with its associated anisotropic elasticae are shown in Figure 11. As before, these anisotropic elasticae are obtained using the integral expression (8).

\section{CONCLUSION}

We have shown that, assuming the Wulff shape of the functional has the right symmetry, all types of elasticae found by Euler have an analogue in the anisotropic case. In addition, images of these curves are easily accessible via computer graphics.

\section{CONFLICTS OF INTEREST}

The authors declare they have no conflicts of interest.

\section{ACKNOWLEDGMENTS}

The second author has been partially supported by MINECOFEDER grant PGC2018-098409-B-100, Gobierno Vasco grant IT1094-16 and by Programa Posdoctoral del Gobierno Vasco, 2018. He would also like to thank the Department of Mathematics and Statistics of Idaho State University for its warm hospitality.

\section{REFERENCES}

[1] Palmer B, Pámpano Á. Anisotropic bending energies of curves. Ann Glob Anal Geom 2020.

[2] Euler L. De curvis elasticis. In: Methodus Inveniendi Lineas Curvas Maximi Minimive Proprietate Gaudentes, Sive Solutio Problematis Isoperimetrici Lattissimo Sensu Accepti. Additamentum 1 Ser. 1, 24. Lausanne; 1744.

[3] Oldfather WA, Ellis CA, Brown DM. Leonhard Euler's elastic curves. Isis 1933;20;72-160.

[4] Wulff G. Zur frage der geschwindigkeit des wachsthums und der auflösung der krystallflächen. Z Kristallogr Crystal Mater 1901;34;449-530.

[5] Gielis J. A generic geometric transformation that unifies a wide range of natural and abstract shapes. Am J Bot 2003;90;333-8.

[6] Gielis J. Inventing the circle: the geometry of nature. Geniaal Press; 2003. 